OPEN ACCESS

Edited by:

Chang Zou,

Jinan University, China

Reviewed by:

Juan Cen,

Henan University, China

Nie Ru-qiong,

Sun Yat-sen University, China

${ }^{*}$ Correspondence:

Yuejiao Zhong

zhongyuejiao1977@126.com

Specialty section:

This article was submitted to Pharmacology of Anti-Cancer Drugs,

a section of the journal

Frontiers in Oncology

Received: 02 September 2021 Accepted: 01 December 2021 Published: 10 January 2022

Citation:

Sheng W, Zhou W, Cao Y and Zhong Y (2022) Revealing the Role of IncRNA CCDC144NL-AS1 and LINC01614 in

Gastric Cancer via Integrative

Bioinformatics Analysis and

Experimental Validation.

Front. Oncol. 11:769563.

doi: 10.3389/fonc.2021.769563

\section{Revealing the Role of IncRNA CCDC144NL-AS1 and LINC01614 in Gastric Cancer via Integrative Bioinformatics Analysis and Experimental Validation}

\author{
Weiwei Sheng ${ }^{1}$, Weihong Zhou ${ }^{1}$, Yundi $\mathrm{Cao}^{2}$ and Yuejiao Zhong $^{3 *}$ \\ 1 Physical Examination Center, Nanjing Drum Tower Hospital, The Affiliated Hospital of Nanjing University Medical School, \\ Nanjing, China, 2 Department of Oncology, Affiliated Taikang Xianlin Drum Tower Hospital, Medical School of Nanjing \\ University, Nanjing, China, ${ }^{3}$ Department of Medical Oncology, Jiangsu Cancer Hospital and Jiangsu Institute of Cancer \\ Research and The Affiliated Cancer Hospital of Nanjing Medical University, Nanjing, China
}

Long non-coding RNAs (IncRNAs) are key regulators in the pathophysiology of gastric cancer, and IncRNAs have been regarded as potential biomarkers and therapeutic targets for gastric cancer. The present study performed the WGCNA analysis of the GSE70880 dataset and aimed to identify novel IncRNAs associated with gastric cancer progression. Based on the WGCNA, the IncRNAs and mRNA co-expression network were constructed. A total of four modules were identified and the eigengenes in different modules were involved in various key signaling pathways. Furthermore, the co-expression networks were constructed between the IncRNAs and mRNA; this leads to the identification of 6 modules, which participated in various cellular pathways. The survival analysis showed that high expression of CCDC144NL antisense RNA 1 (CCDC144NLAS1) and LINC01614 was positively correlated with the poor prognosis of patients with gastric cancer. The in vitro validation results showed that CCDC144NL-AS1 and LINC01614 were both up-regulated in the gastric cancer cells. Silence of CCDC144NLAS1 and LINC01614 both significantly suppressed the cell proliferation and migration of gastric cancer cells, and also promoted the chemosensitivity of gastric cancer cells to 5fluorouracil. Collectively, our results suggested that the newly identified two IncRNAs (CCDC144NL-AS1 and LINC01614) may act as oncogenes in gastric cancer.

Keywords: IncRNAs, WGCNA, gastric cancer, CCDC144NL-AS1, LINC01614, prognosis

\section{INTRODUCTION}

Gastric cancer represents one of the most common human malignancies, and the occurrence of gastric cancer is region dependent with about $60 \%$ of the cases are found in developing regions (1, 2 ). With the improved living condition and early screening, the new cases of gastric cancer in these regions are decreasing. However, the prognosis of gastric cancer has not been largely improved. For the treatment of gastric cancer surgical resection is the key treatment for gastric cancer, while the 
five-year overall survival of gastric cancer patients after surgical treatment depends on the tumor stages with $\sim 90 \%$ in early-stage and $\sim 20 \%$ in advanced stage gastric cancer patients (3). In these patients diagnosed at an advanced stage, chemotherapy instead of surgical resection is considered for alleviating the symptoms in patients, however, chemotherapy only exhibits a modest beneficial effect on patients with metastatic patients, and chemotherapy has been largely limited chemoresistance $(4,5)$. In this regard, further exploration into the mechanisms underlying gastric cancer progression may be helpful for us to develop novel strategies, to improve the clinical outcomes of gastric cancer patients.

Long non-coding RNAs (lncRNAs) belong to a type of RNA without protein-coding capacity, and lncRNAs are longer than 200 nucleotides in length (6). In the past decade, lncRNA has been extensively examined in various diseases including cancers, due to its diverse biological functions such as regulating cell proliferation, apoptosis, invasion, modulating immunity, and so on (7). In gastric cancer, lncRNAs such as HOX transcript antisense RNA, plasmacytoma variant translocation 1, nuclear enriched abundant transcript 1 , maternally expressed 3 and colon cancer associated transcript 1 have been reported to involve in gastric cancer progression and the prognosis of patients with gastric cancer (8-12). Due to the large number of existing lncRNAs in the human genome, more efforts are needed to discover more lncRNAs in regulating the pathophysiology of gastric cancer. Recently, high-throughput screening technology such as RNA-sequencing has been performed to identify novel lncRNAs. In addition, the analysis of high-throughput datasets using different bioinformatic strategies has enabled us to identify lncRNAs more efficiently. For example, Zhao et al., performed the analysis in the Gene Expression Omnibus (GEO) datasets including GSE58828 and GSE27342 and found that LINC00355, a novel lncRNA, induced gastric cancer progression via enhancing ubiquitination of p53 (13). Ren et al., performed the weighted gene co-expression network analysis (WGCNA), and the analysis revealed ILF3-AS1 acted as a ceRNA to regulate polypyrimidine tract binding protein 1 by repressing miR-29a expression in gastric cancer (14). Foroughi et al., performed the comprehensive bioinformatic analysis and revealed the tissue-specific down-regulation of prostate cancer associated transcript 18 and LINC01133 in gastric cancer development (15). The role of lncRNAs in the drug resistance of gastric cancer cells has also been demonstrated in various studies. For examples, He et al., showed that mesenchymal stem cells- regulated IncRNA MACC1-AS1enhanced chemoresistance through fatty acid oxidation in gastric cancer (16). Zhang et al., found that lncRNA colorectal neoplasia differentially expressed attenuates chemoresistance in gastric cancer via serine and arginine rich splicing factor 6 -regulated alternative splicing of phosphatidylinositol binding clathrin assembly protein (17).

Given the promising role of lncRNAs in gastric development, the present study was undertaken to discover novel lncRNAs correlated with the progression of gastric cancer. In this study, we performed the WGCNA analysis using the GEO dataset GSE70880 and constructed the IncRNAs co-expression network to identify the hub lncRNAs. In this dataset, the expression profiles of cancer and adjacent normal tissues form 76 patients (20 with gastric cancer, 20 with colon cancer, 16 with liver cancer and 20 with lung cancer) were studied by microarray and a set of lncRNAs as well as mRNAs were identified as potential biomarkers general to different types of cancer (18-20). In addition, the identified novel hub lncRNAs were subjected to in vitro validation studies. The present study will further advance our understanding of the role of the pathophysiology of gastric cancer.

\section{MATERIALS AND METHODS}

\section{Selection of Expression Datasets From GEO Database}

The GSE70880 dataset was obtained from the GEO database (19). The dataset contained 20 gastric cancer tissue samples and 20 adjacent normal gastric tissue samples. The quality control of the raw data was analyzed using an array Quality package and the expression datasets were further analyzed using the limma package in $\mathrm{R}$ software. For criteria for significantly differentially expressed genes were set at false discovery rate (FDR) $<0.05$ and fold changes $(\mathrm{FCs})>2$.

\section{WGCNA}

The WGCNA package in the $\mathrm{R}$ software was utilized for the analysis of lncRNA mRNA co-expression modules $(14,21,22)$. The WGCNA parameters of soft threshold power of the adjacency matrix and the criteria of correlation coefficient square of eigengenes were defined according to the approximate scale-free topology preconditions and the criteria of cut-off of $\geq 30$ genes and cut height $=0.15$. The adjacency matrix dissimilarity was 0.2 . Then, the WGCNA modules (coexpression network) of eigengenes were identified and the networks correlated with agronomic traits were identified with the criterion of stability correlation $\mathrm{p} \leq 0.05$.

\section{Functional Enrichment Analysis}

The differentially expressed genes from the analyzed dataset were separately subjected to the enrichment analysis for Gene Ontology (GO; http://www.Geneontology.org/) and KEGG (Kyoto Encyclopedia of Genes and Genomes) pathways. Significant GO and KEGG pathways were identified with the criterion of $\mathrm{p}<0.05$.

\section{Survival Analysis of the Patients With Gastric Cancer}

For the effects of the lncRNAs on the survival of gastric cancer patients, the Kaplan-Meier analysis was performed by using The Cancer Genome Atlas database. In the database, a total of 352 patients with gastric cancer were included. $\mathrm{P}<0.05$ was considered statistically significant. 


\section{Cell Culture}

The gastric cancer cells including AGS and SGC7901 and the human normal gastric epithelial cells (GES-1) were obtained from the Chinese Academy of Sciences (Shanghai, China). The cells were cultured in the RPMI-1640 medium (Sigma-Aldrich, St. Louis, USA) supplemented with $100 \mathrm{mg} / \mathrm{ml}$ streptomycin, $10 \%$ fetal bovine serum and $100 \mathrm{U} / \mathrm{ml}$ penicillin (SigmaAldrich). The cells were maintained in a humidified incubator with $5 \% \mathrm{CO}_{2}$ at $37^{\circ} \mathrm{C}$. For the establishment of 5 -fluorouracil (5-FU)-resistant SGC7901 cells, the SGC7901/5-FU cell line was generated by using a habitual stepwise method, according to the previous studies (23).

\section{Design and Synthesis of siRNAs, Cell Transfections With siRNAs}

The respective siRNAs for CCDC144NL antisense RNA 1 (CCDC144NL-AS1) and LINC01614 were designed and synthesized by RiboBio (Guangzhou, China), and respective scrambled siRNAs were served as negative controls (NCs). For the cell transfections, the cells were seeded at $1 \times 10^{6}$ cells/well. After cells reached $\sim 80 \%$ confluence, cells were transfected with $30 \mathrm{nM}$ respective siRNAs using Lipofectamine Plus Reagent (Invitrogen, Carlsbad, USA) as per the manufacturer's protocol.

\section{Quantitative Real-Time PCR (qRT-PCR)}

The RNA was extracted from cells by using the Trizol reagent (Sigma-Aldrich) as per the manufacturer's protocol. The PrimeScript RT reagent Kit (Takara, Dalian, China) was used to reversely transcribe mRNA into cDNA. The real-time PCR was performed on an ABI7500 Real-Time PCR System (Applied Biosystems, Waltham, USA) by using the SYRB Premxi DimerEraser Kit (Takara). The relative expression of the lncRNAs in the cells was normalized to GAPDH and was calculated by using the comparative $\mathrm{Ct}$ method.

\section{Cell Counting Kit-8 (CCK-8) Assay}

The CCK- 8 assay was determined by using the CCK- 8 assay kit (Beyotime, Beijing, China) as per the manufacturer's protocol. After $0,24,48,72 \mathrm{~h}$ siRNA transfections, the proliferation of gastric cancer cells were incubated with CCK- 8 reagent, and the cell proliferative index was determined by measuring the absorbance at a wavelength of $450 \mathrm{~nm}$.

\section{Wound Healing Assay}

Cell migration ability was measured using the wound healing assay. Gastric cancer cells were plated into 6-well plates at a density of $1 \times 10^{6}$ cells/well and cultured until $90 \%$ confluence. A micropipette tip was then used to make a perpendicular scratch in the middle of each well. After incubation for $24 \mathrm{~h}$, images were taken and the wound-healing rate was assessed by ImageJ.

\section{Determination of Chemosensitivity of SGC7901/5-FU Cells to 5-FU}

For the determination of chemosensitivity, cells were seeded at $1 \times 10^{5}$ cells/well. After that, the cells were incubated with varying concentrations of 5-FU. At $48 \mathrm{~h}$ after 5-FU treatment, the cell proliferative index was determined by CCK- 8 assay as per the manufacturer's protocol.

\section{Statistical Analysis}

For the in vitro experiments, all the experiments were performed in triplicate. The results were shown as mean \pm standard error of the mean. GraphPad Prism software was used to perform the statistical analysis. The differences between different treatment groups were determined by unpaired t-test or one-way analysis of variance followed by Bonferroni's post-hoc test. $\mathrm{P}<0.05$ was considered statistically significant.

\section{RESULTS}

\section{Volcano Plot of Differentially Expressed mRNA and IncRNA in Gastric Cancer}

Based on the analysis, we performed the bioinformatics analysis, and identified a series of differentially expressed mRNAs (129 upregulated and 187 downregulated) and differentially expressed lncRNAs (19 upregulated and 52 downregulated) between gastric cancer and adjacent normal gastric tissues (Figures 1A, B).

\section{GSEA GO and GSEA KEGG Pathway Analysis of Differentially Expressed mRNA and IncRNA}

The differentially expressed mRNAs and lncRNAs were further subjected to GSEA GO and KEGG analysis. In the GSEA GO analysis, the mRNA and lncRNAs were mainly enriched in "mitotic cell cycle", "regulation of mitotic cell cycle", "mitotic cell cycle process", "cell cycle", "nuclear division", "chromosome" and so on (Figure 2A). In the GSEA KEGG analysis, the mRNAs and lncRNAs were mainly enriched in "Pathways of neurodegeneration-multiple diseases", "cell adhesion molecules", "neuroactive ligand-receptor interaction", "human papillomavirus infection", "glutamatergic synapse" and so on (Figure 2B).

\section{WGCNA of GSE70880}

Weighted gene co-expression network analysis is a systems biology method to understand correlation patterns among genes across different samples. WGCNA can be used to find clusters or modules. Based on the WGCNA analysis, a total of 5 co-expressed modules are in the analyzed dataset (Figures 3AC). Subsequently, the association between each of the modules and clinical traits (cancer vs normal) was further explored. Based on the analysis, the blue and turquoise modules were strongly correlated with the occurrence of gastric cancer (Figure 3D). The differentially expressed lncRNAs in different modules were clustered between the gastric cancer group and the normal gastric tissue group (Figure 4). Furthermore, the co-expression network was constructed based on the WGCNA analysis, and the constructed network was demonstrated in Figure 5. In addition, Figure 5 illustrated the hub genes in different modules from the WGCNA analysis. 

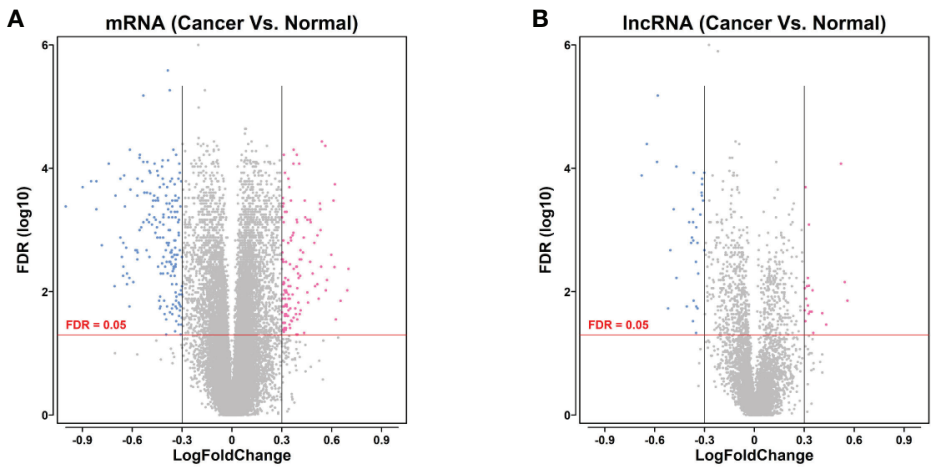

FIGURE 1 | Volcano plot of differentially expressed mRNA and IncRNA in gastric cancer. (A) Volcano plot of differentially expressed mRNA of the gastric cancer tissues between gastric cancer group and non-cancerous group. (B) Volcano plot of differentially expressed IncRNA of the gastric cancer tissues between gastric cancer group and non-cancerous group. mRNAs or IncRNAs with $\log _{2} \mathrm{FC}>2$ and FDR $<0.05$ were shown in red dots; $m R N A s$ or $\operatorname{IncRNAs~with~} \log _{2} \mathrm{FC}<-2$ and FDR $<0.05$ were in blue dots. Grey dots represent the non-differentially expressed genes.

A

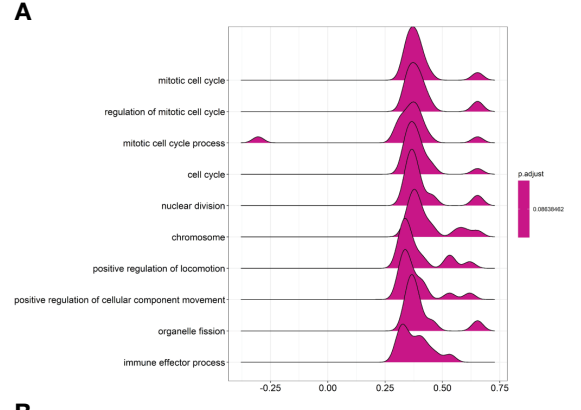

B

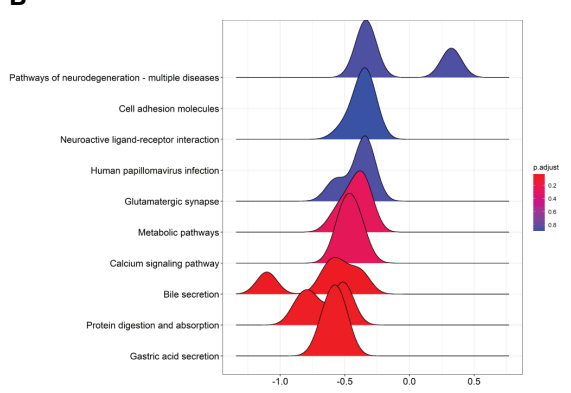

FIGURE 2 | GSEA GO and GSEA KEGG pathway analysis of differentially expressed mRNA and IncRNA. (A) Joyplot of GSEA GO analysis. (B) Joyplot of GSEA KEGG pathway analysis.

\section{Functional Enrichment Analysis of Differentially Expressed Genes in the Different Modules}

The functional enrichment analysis was further performed in the genes from different modules. For the turquoise module, the genes were mainly enriched in "collagen catabolic process", "positive regulation of lipid localization", "extracellular matrix disassembly", "negative regulation of hormone secretion" and "hormone secretion" (Figure 6). For the blue module, the genes were mainly enriched in "vascular process in circulatory system", "neutral lipid catabolic process", "negative regulation of fatty acid oxidation", "gastric acid secretion" and "glycerolipid catabolic process" (Figure 6). For the brown module, the genes were mainly enriched in "prostate gland morphogenesis", "prostate gland development", "positive regulation of mitotic nuclear division", "positive regulation of nuclear division" and "positive regulation of protein localization to plasma membrane". For the yellow module, the genes were mainly enriched in "keratan sulfate catabolic process", "muscle system process", "regulation of cell growth", positive regulation of cell growth" and "tissue remodeling".

\section{Kaplan-Meier Analysis for the Effects of the IncRNAs on the Survival of Gastric Cancer Patients}

Furthermore, the effects of two lncRNAs (CCDC144NL-AS1 and LINC01614) on the survival of gastric cancer patients were evaluated by Kaplan-Meier analysis. The survival analysis included a total of 352 patients, and high expression of CCDC144NL-AS1 and LINC01614 are both associated with shorter overall survival of patients with gastric cancer (Figures 7A, B).

\section{Effects of CCDC144NL-AS1 and LINC01614 Silence on the Proliferation, Migration and} Chemosensitivity of Gastric Cancer Cells

The qPCR assay was performed to confirm the expression of CCDC144NL-AS1 and LINC01614 in gastric cancer cells, and CCDC144NL-AS1 and LINC01614 were significantly upregulated in AGS and SGC7901 cells compared to that in GES-1 cells (Figures 8A, B). The knockdown of CCDC144NL-AS1 and LINC01614 in AGS and SGC7901 cells was achieved by transfecting these cells with respective siRNAs for CCDC144NL- 

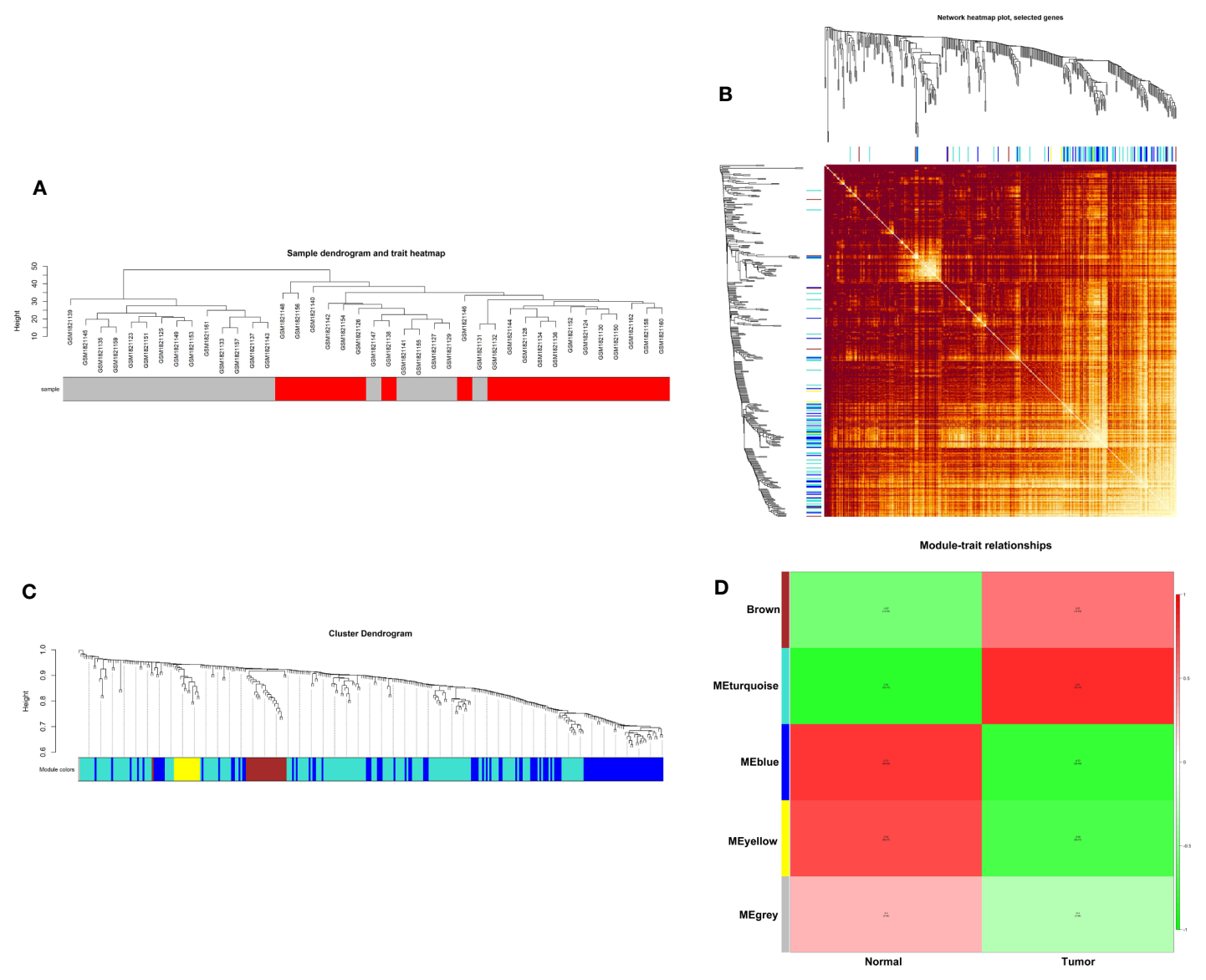

FIGURE 3 | WGCNA of GSE70880. (A) Hierarchical clustering dendrogram of the samples. (B) Heatmap representing the Topological Overlap Matrix (TOM) among all genes in the WGCNA. (C) Average linkage hierarchical clustering dendrogram of the genes. The input was the topological overlap-based dissimilarity. Modules, designated by color code, are the branches of the clustering tree. (D) Correlation of module eigengenes to clinical and pathological traits.

AS1 and LINC01614 (Figures 8C, D). The CCK-8 assay showed that the silence of CCDC144NL-AS1 and LINC01614 silence both significantly repressed the proliferation of AGS and SGC7901 cells (Figure 8E-H). Furthermore, the knockdown of CCDC144NLAS1 and LINC01614 both impaired the wound closure capacity of AGS and SGC7901 cells (Figures 9A, B). The effects of the lncRNAs on the chemosensitivity of gastric cancer cells to 5-FU were determined in SGC7901/5-FU cells. Silence of CCDC144NLAS1 and LINC01614 both significantly reduced the IC50 values for 5-FU in the SGC7901/5-FU cells (Figures 9C-F).

\section{DISCUSSION}

LncRNAs are key regulators in the pathophysiology of gastric cancer, and lncRNAs have been regarded as potential biomarkers and therapeutic targets for gastric cancer (24). With the great advancement in high-throughput technologies, bioinformatic exploration has enabled us to efficiently identify novel lncRNAs associated with cancer progression. In this study, we performed the WGCNA analysis of the GEO dataset (GSE70880), and our results revealed the lncRNA coexpression network. In addition, several hub lncRNAs were detected in the co-expression network based on the WGCNA analysis. The survival analysis showed that high expression of CCDC144NL-AS1 and LINC01614 was positively correlated with the poor prognosis of patients with gastric cancer. The in vitro validation results showed that CCDC144NL-AS1 and LINC01614 were both up-regulated in the gastric cancer cells. Silence of CCDC144NL-AS1 and LINC01614 both significantly suppressed the cell proliferation and migration of gastric cancer cells, and also promoted the chemosensitivity of gastric cancer cells to cisplatin. Collectively, our results suggested that the newly identified two lncRNAs (CCDC144NL-AS1 and LINC01614) may act as oncogenes in gastric cancer.

WGCNA is a system biology strategy to reveal correlation patterns among genes across different samples. This analysis is useful in identifying the modules or clusters. In addition, the eigengene can be used to summarize modules, and examine the correlation between the modules and sample traits. Le et al., performed the WGCNA using the GSE76250 dataset and 


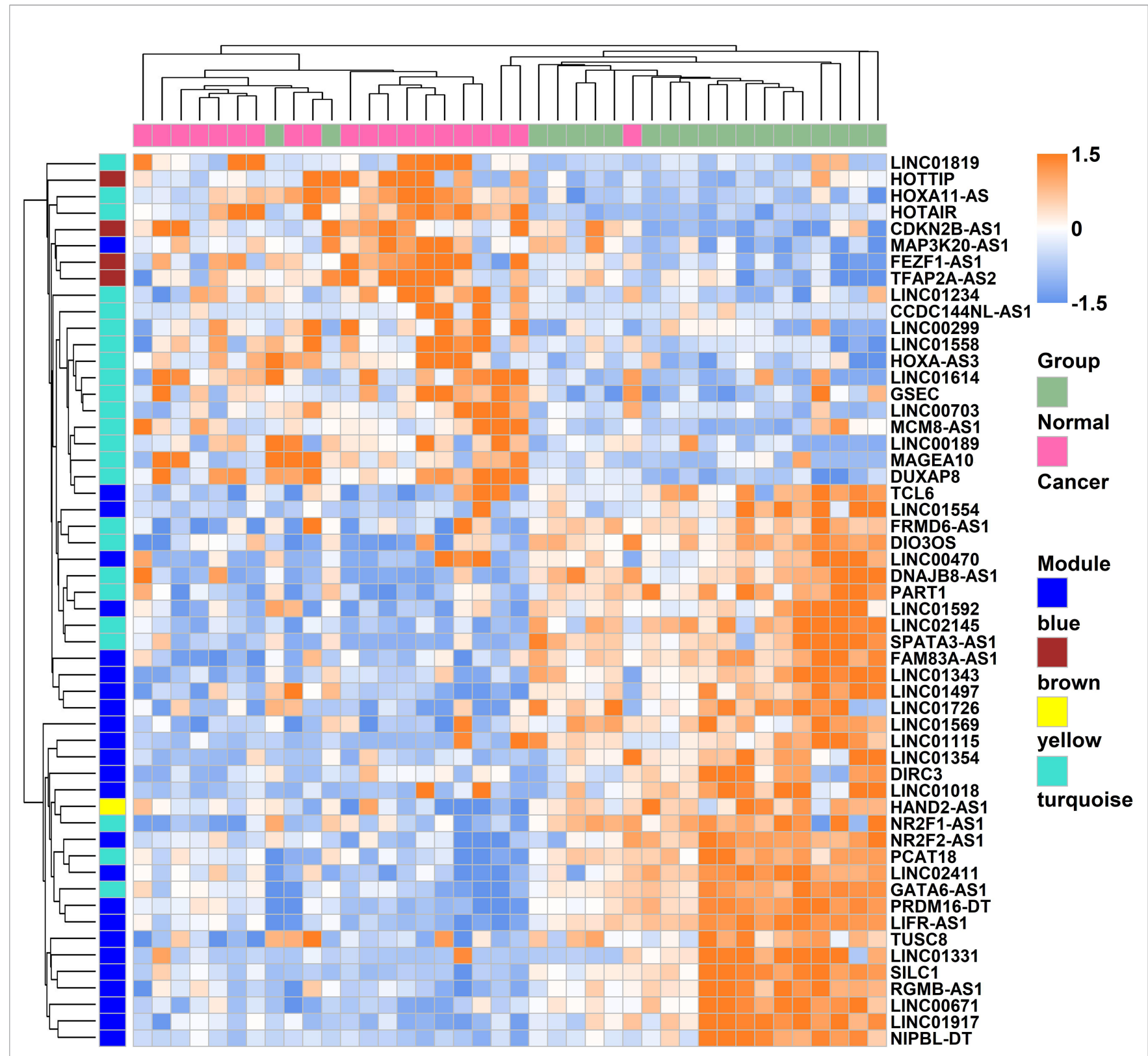

FIGURE 4 | Heatmap of the IncRNAs which were involved in the modules expressed between gastric cancer and normal gastric tissues.

revealed a novel competing endogenous RNA network for triplenegative breast cancer (25). Qian et al, performed the analysis of lncRNA-mRNA networks after MEK1/2 inhibition based on WGCNA in pancreatic cancer and found that NONHSAT185150.1 and beta-1,4-galactosyltransferase were negatively correlated with MEK1/2 (26). Consistently, Giulietti et al., performed a similar analysis and revealed novel biomarkers (LINC00675 and LINC01133) for pancreatic cancer (21). Based on the WGCNA analysis, Li et al., demonstrated that lncRNA51663 and FLJ46906 were remarkably increased in $\mathrm{H}$. pylori-infected cells and consistently overexpressed in human gastric cancer tissues compared to adjacent normal tissues (27). In agreement with previous studies, we performed the WGCNA on the GSE70880 dataset and identified two novel lncRNAs (CCDC144NL-AS1 and LINC01614) that may be associated with the survival of patients with gastric cancer.

The role of CCDC144NL-AS1 has been implicated in several types of cancers. Zhang et al., showed that knockdown of CCDC144NL-AS1 attenuated migration and invasion phenotypes in endometrial stromal cells from endometriosis (28). CCDC144NL-AS1 also promoted the oncogenicity of osteosarcoma by acting as a molecular sponge for microRNA490-3p and thereby increasing HMGA2 Expression (29). In addition, Zhang found that CCDC144NL-AS1 could promote the development of hepatocellular carcinoma by inducing WD repeat domain 5 expression via sponging miR- 


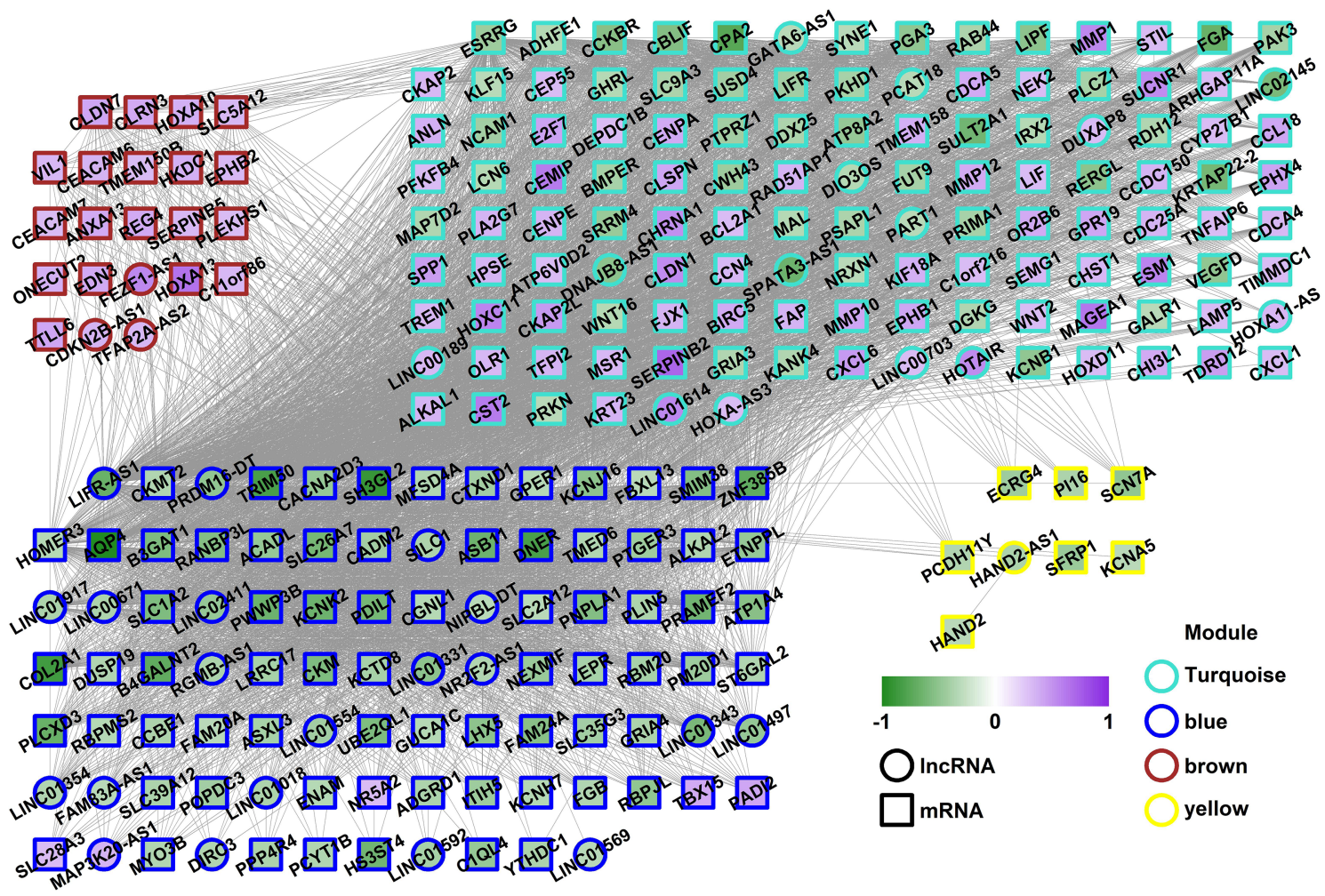

FIGURE 5 | Co-expression network in the different modules. The fill color showed the log2FC of each gene; the border color showed the module the gene was from; the shape showed the type of gene. The square indicates the mRNA and the circle indicates the IncRNA.

940 (30). A recent study demonstrated that CDC144NL-AS1 served as a prognosis biomarker for non-small cell lung cancer and promoted cellular function by targeting miR-490-3p (31). However, the role of CDC144NL-AS1 in gastric cancer progression remains unknown. Our in vitro studies showed that silence of CDC144NL-AS1 inhibited the progression of gastric cancer cells and enhanced the chemosensitivity of gastric cancer cells to cisplatin, suggesting that CDC144NLAS1 may serve as an oncogenic RNA in gastric cancer.

The functional role of LINC01614 in cancer progression was first reported by Liu et al., and the study found that LINC01614 suppressed lung cancer cell progression by regulating miR-217 and down-regulating forkhead box P1 (32). Vishnubalaji et al., performed the lncRNA transcriptional analysis in breast cancer and identifies LINC01614 as a non-favorable prognostic biomarker regulated by transforming growth factor beta and focal adhesion kinase signaling (33). Consistently, Wang et al., found that LINC01614 could serve as a potential biomarker for prognostic prediction in breast cancer (34). In glioma, Wang et al., found that SP1-mediated upregulation of LINC01614 functions a ceRNA for miR-383 to facilitate tumor progression via regulation of ADAM12 (35). Recently, Cai et al., showed that LINC01614 promoted osteosarcoma progression via the miR520a-3p/sorting nexin 3 axis (36). Our in vitro studies showed that silence of LINC01614 attenuated the progression of gastric cancer cells and enhanced the chemosensitivity of gastric cancer cells to cisplatin, suggesting that LINC01614 may serve as an oncogenic RNA in gastric cancer.

This study has several limitations for consideration. Firstly, the WGCNA was performed in only one dataset, and future studies may consider analyzing more datasets to reveal more novel lncRNAs associated with gastric cancer progression. Secondly, the prognostic role of the newly identified lncRNAs has not been examined in the clinical studies, which should be explored in future studies. Thirdly, the investigation into the mechanistic role of CCDC144NL-AS1 and LINC01614 is still at the early stage, and more mechanistic studies should be considered, to reveal the potential actions of these two lncRNAs in gastric cancer.

\section{CONCLUSIONS}

In conclusion, our study performed the WGCNA and revealed that CCDC144NL-AS1 and LINC01614 might be potential biomarkers for the prognosis of gastric cancer patients. Further in vitro functional studies indicated that CCDC144NLAS1 and LINC01614 might serve as oncogenic lncRNAs in gastric cancer. The present study for the first time provides novel insights into the role of CCDC144NL-AS1 and LINC01614 in the pathophysiology of gastric cancer. 


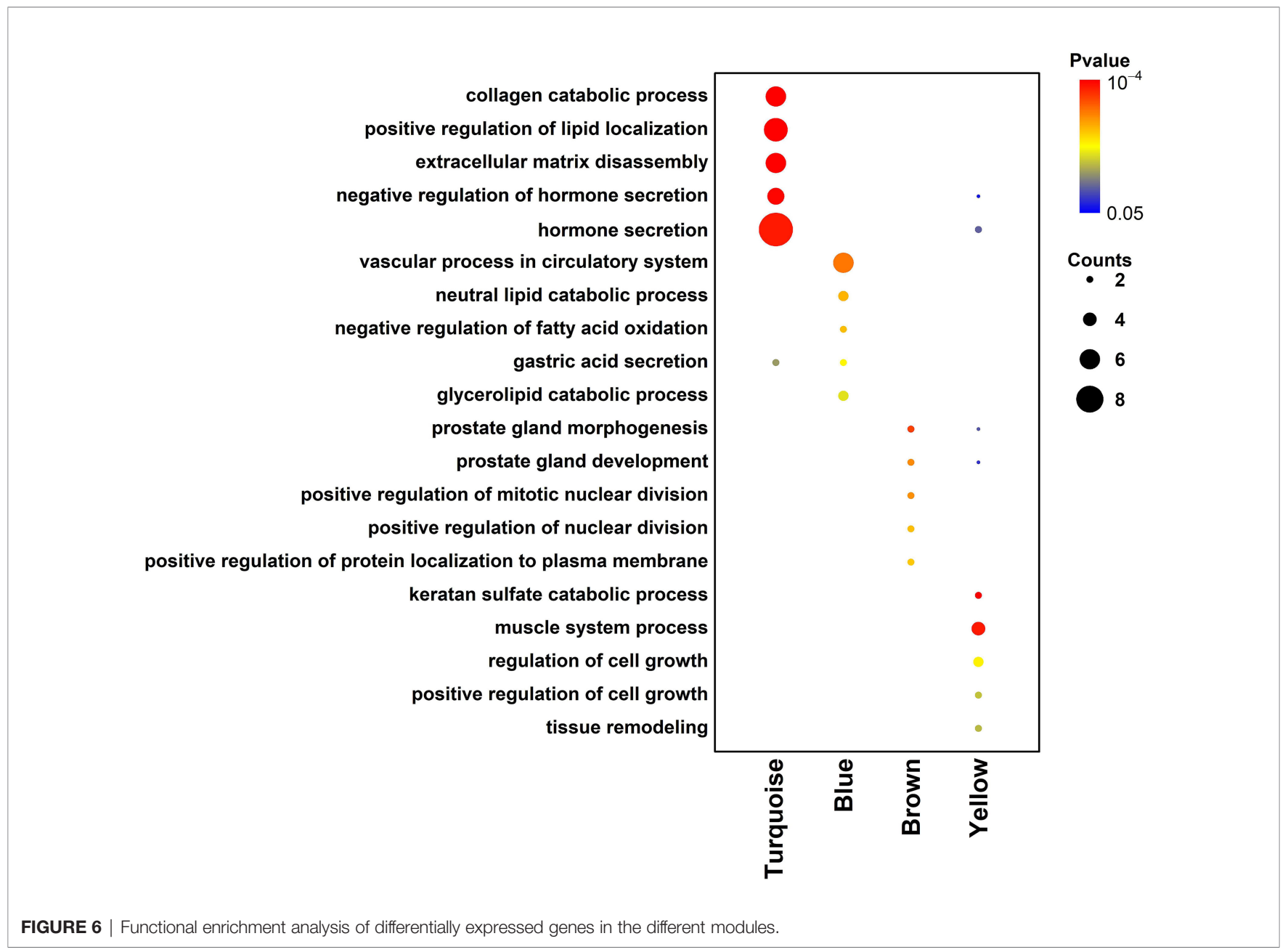

A

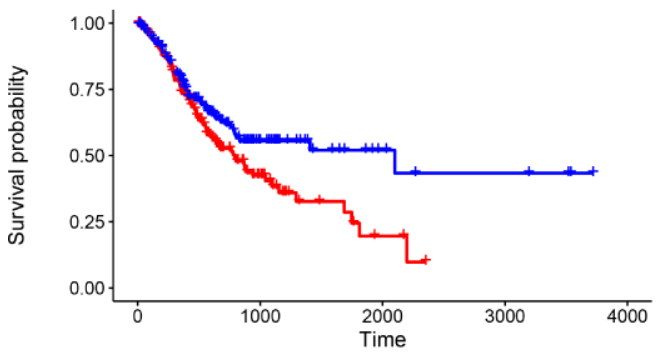

Number at risk

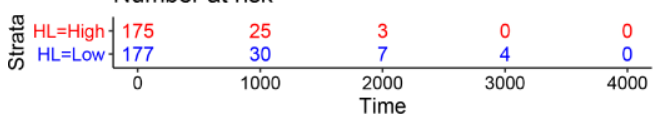

B

LINC01614 $p=0.011$

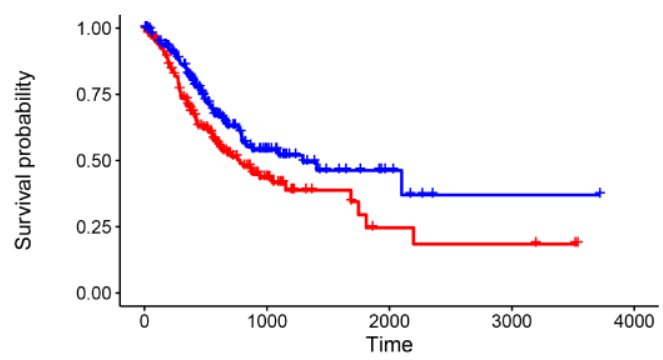

Number at risk

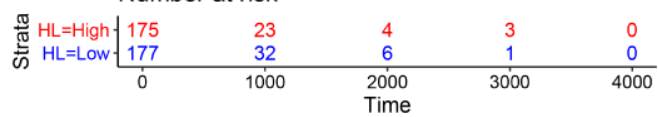

FIGURE 7 | Kaplan-Meier analysis for the effects of the IncRNAs on the survival of gastric cancer patients. Kaplan-Meier survival analysis for (A) CCDC144NL-AS1 and (B) LINC01614 in gastric cancer patients. 
A

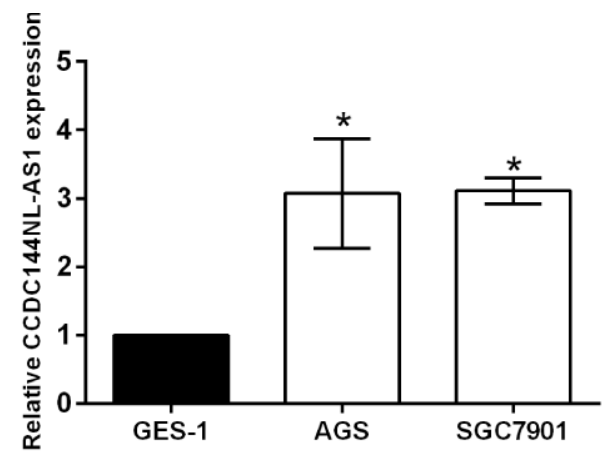

c

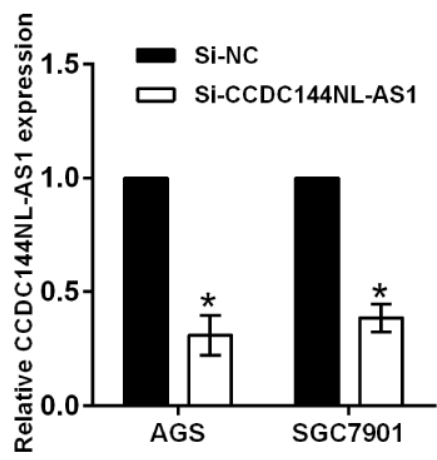

E

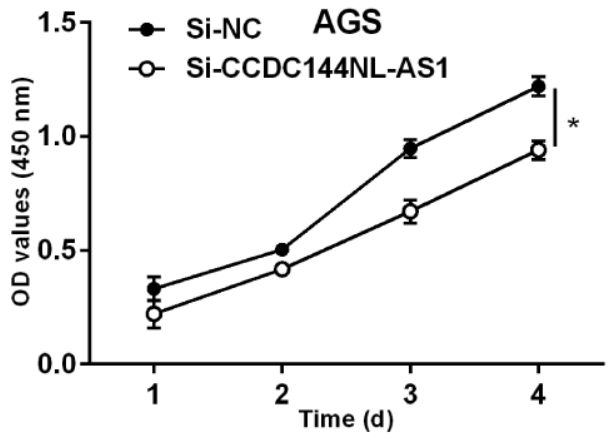

G

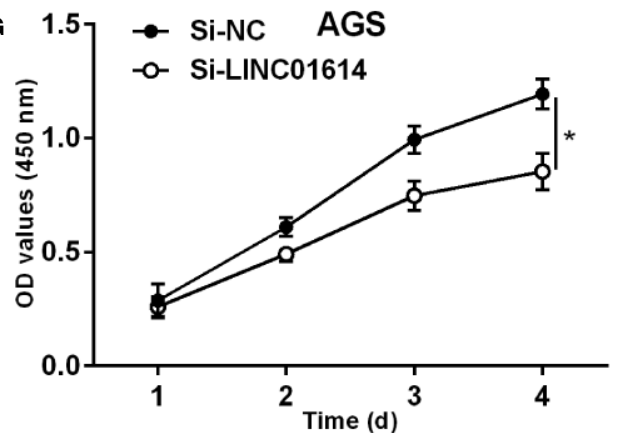

B

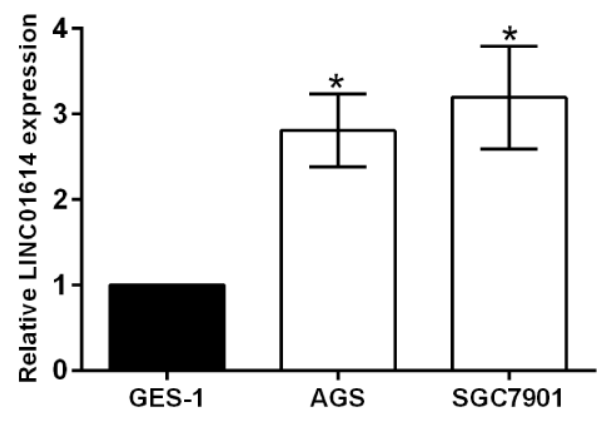

D

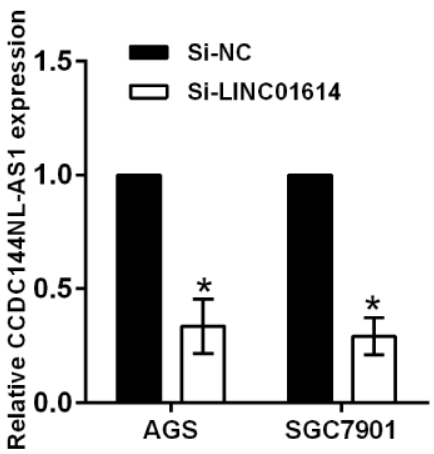

$\mathbf{F}$

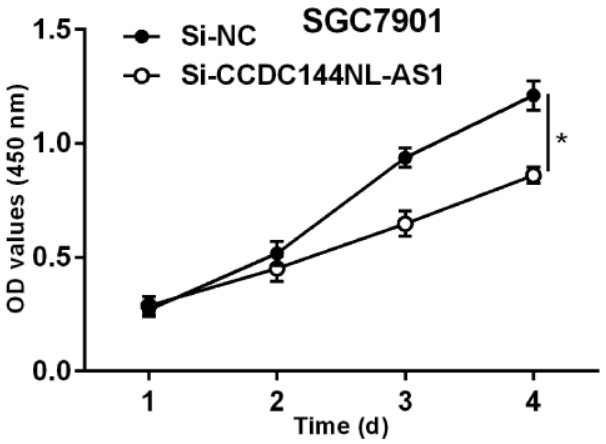

H

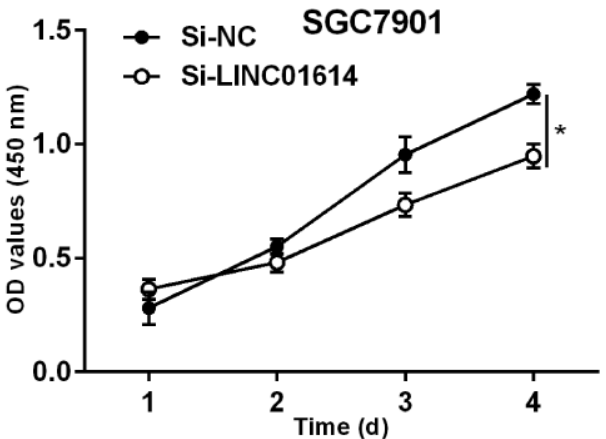

FIGURE 8 | Effects of CCDC144NL-AS1 and LINC01614 silence on gastric cancer cell progression. (A) Expression of CCDC144NL-AS1 in GES-1, AGS and SGC7901 cells. (B) Expression of LINC01614 in GES-1, AGS and SGC7901 cells. (C) Expression of CCDC144NL-AS1 in AGS and SGC7901 cells after siRNAs transfections. (D) Expression of LINC01614 in AGS and SGC7901 cells after siRNAs transfections. (E, F) Effects of CCDC144NL-AS1 silence on the proliferation of AGS and SGC7901 cells. (G, H) Effects of LINC01614 silence on the proliferation of AGS and SGC7901 cells. N = 3. Significant between treatment groups were indicated as ${ }^{*} \mathrm{p}<0.05$. 
A

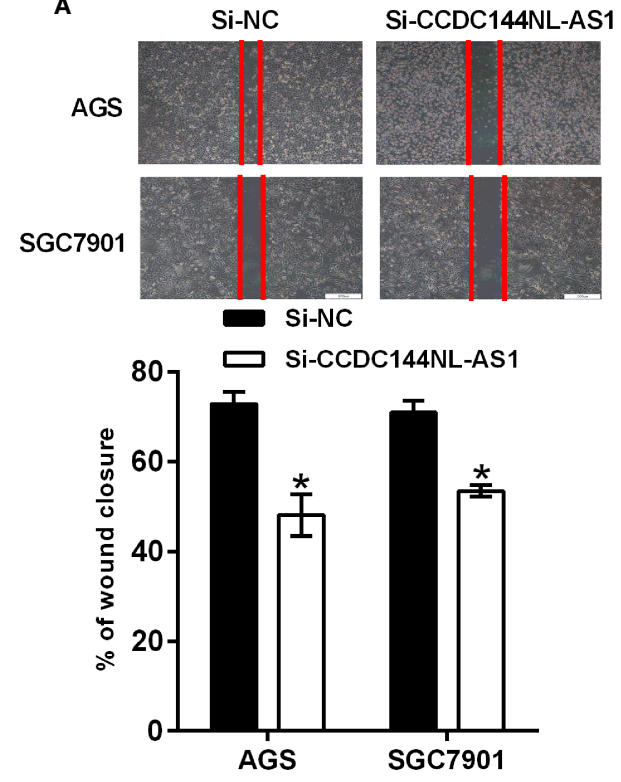

C

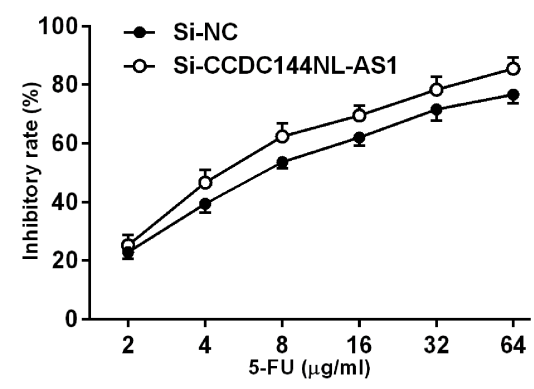

E

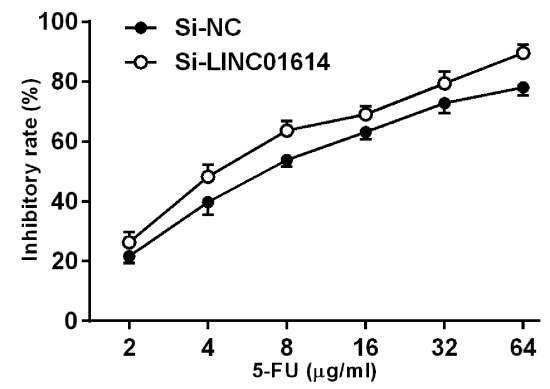

B

Si-NC Si-LINC01614

AGS
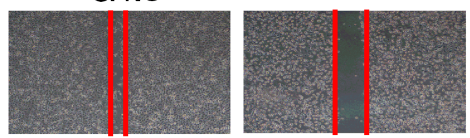

SGC7901
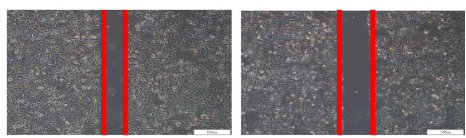

- Si-NC

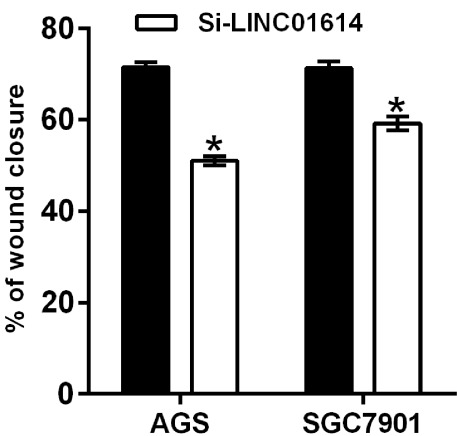

D

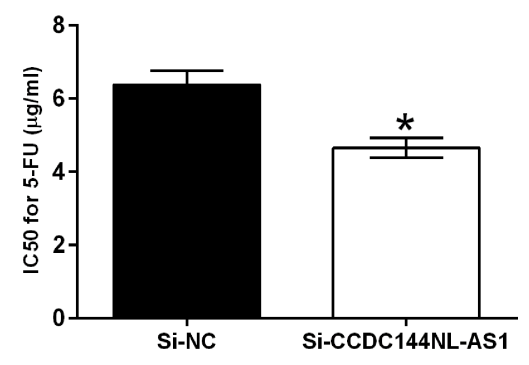

$\mathbf{F}$

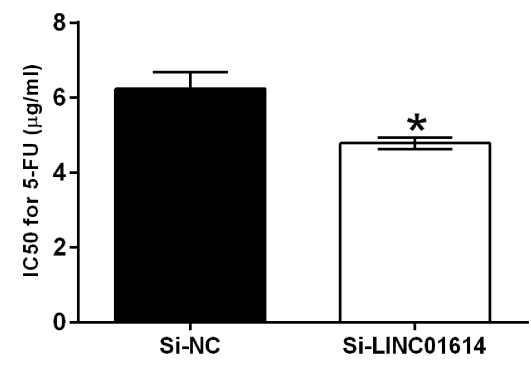

FIGURE 9 | Effects of CCDC144NL-AS1 and LINC01614 silence on the migration and chemosensitivity of gastric cancer cells. (A) Effects of CCDC144NL-AS1 silence on the migration of AGS and SGC7901 cells. Top panel shows the representative images of the wound healing area at $24 \mathrm{~h}$ after wound scratching. (B) Effects of LINC01614 silence on the migration of AGS and SGC7901 cells. Top panel shows the representative images of the wound healing area at $24 \mathrm{~h}$ after wound scratching. (C, D) Effects of CCDC144NL-AS1 silence on the chemosensitivity of SGC7901/5-FU cells to 5-FU. (E, F) Effects of LINC01614 silence on the chemosensitivity of SGC7901/5-FU cells to 5 -FU. $N=3$. Significant between treatment groups were indicated as ${ }^{*} p<0.05$. 


\section{DATA AVAILABILITY STATEMENT}

The original contributions presented in the study are included in the article/supplementary material. Further inquiries can be directed to the corresponding author.

\section{AUTHOR CONTRIBUTIONS}

YZ designed and supervised the whole project. WS and WZ performed the experiments and summarized the data. YZ

\section{REFERENCES}

1. Shah SC. Gastric Cancer: A Neglected Threat to Racial and Ethnic Minorities in the USA. Lancet Gastroenterol Hepatol (2021) 6:266-7. doi: 10.1016/S24681253(21)00064-9

2. Ward ZJ, Scott AM, Hricak H, Atun R. Global Costs, Health Benefits, and Economic Benefits of Scaling Up Treatment and Imaging Modalities for Survival of 11 Cancers: A Simulation-Based Analysis. Lancet Oncol (2021) 22:341-50. doi: 10.1016/S1470-2045(20)30750-6

3. Smyth EC, Nilsson M, Grabsch HI, Van Grieken NC, Lordick F. Gastric Cancer. Lancet (2020) 396:635-48. doi: 10.1016/S0140-6736(20)31288-5

4. Van Cutsem E, Sagaert X, Topal B, Haustermans K, Prenen H. Gastric Cancer. Lancet (2016) 388:2654-64. doi: 10.1016/S0140-6736(16)30354-3

5. Lott PC, Carvajal-Carmona LG. Resolving Gastric Cancer Aetiology: An Update in Genetic Predisposition. Lancet Gastroenterol Hepatol (2018) 3:87483. doi: 10.1016/S2468-1253(18)30237-1

6. Salta E, De Strooper B. Non-Coding RNAs With Essential Roles in Neurodegenerative Disorders. Lancet Neurol (2012) 11:189-200. doi: 10.1016/ S1474-4422(11)70286-1

7. Chowdhary A, Satagopam V, Schneider R. Long Non-Coding RNAs: Mechanisms, Experimental, and Computational Approaches in Identification, Characterization, and Their Biomarker Potential in Cancer. Front Genet (2021) 12:649619. doi: 10.3389/fgene.2021.649619

8. Al-Rugeebah A, Alanazi M, Parine NR. MEG3: An Oncogenic Long NonCoding RNA in Different Cancers. Pathol Oncol Res (2019) 25:859-74. doi: 10.1007/s12253-019-00614-3

9. Fattahi S, Kosari-Monfared M, Golpour M, Emami Z, Ghasemiyan M, Nouri $M$, et al. LncRNAs as Potential Diagnostic and Prognostic Biomarkers in Gastric Cancer: A Novel Approach to Personalized Medicine. J Cell Physiol (2020) 235:3189-206. doi: 10.1002/jcp.29260

10. Ma P, Pan Y, Yang F, Fang Y, Liu W, Zhao C, et al. KLF5-Modulated lncRNA NEAT1 Contributes to Tumorigenesis by Acting as a Scaffold for BRG1 to Silence GADD45A in Gastric Cancer. Mol Ther Nucleic Acids (2020) 22:38295. doi: 10.1016/j.omtn.2020.09.003

11. Martínez-Barriocanal Á., Arango D, Dopeso H. PVT1 Long Non-Coding RNA in Gastrointestinal Cancer. Front Oncol (2020) 10:38. doi: 10.3389/ fonc. 2020.00038

12. Olesiński T, Lutkowska A, Balcerek A, Sowińska A, Piotrowski P, Trzeciak T, et al. Long Noncoding RNA CCAT1 Rs67085638 SNP Contribution to the Progression of Gastric Cancer in a Polish Population. Sci Rep (2021) 11:15369. doi: 10.1038/s41598-021-94576-9

13. Zhao W, Jin Y, Wu P, Yang J, Chen Y, Yang Q, et al. LINC00355 Induces Gastric Cancer Proliferation and Invasion Through Promoting Ubiquitination of P53. Cell Death Discov (2020) 6:99. doi: 10.1038/s41420-020-00332-9

14. Ren ZH, Shang GP, Wu K, Hu CY, Ji T. WGCNA Co-Expression Network Analysis Reveals ILF3-AS1 Functions as a CeRNA to Regulate PTBP1 Expression by Sponging miR-29a in Gastric Cancer. Front Genet (2020) 11:39. doi: 10.3389/fgene.2020.00039

15. Foroughi K, Amini M, Atashi A, Mahmoodzadeh H, Hamann U, Manoochehri M. Tissue-Specific Down-Regulation of the Long NonCoding RNAs PCAT18 and LINC01133 in Gastric Cancer Development. Int J Mol Sci (2018) 19:3881. doi: 10.3390/ijms19123881 wrote the manuscript. YZ revised the drafted manuscript. All authors contributed to the article and approved the submitted version.

\section{FUNDING}

The work was supported by grants from the Scientific Research Project of Jiangsu Provincial Health Committee (Z2021056).

16. He W, Liang B, Wang C, Li S, Zhao Y, Huang Q, et al. MSC-Regulated lncRNA MACC1-AS1 Promotes Stemness and Chemoresistance Through Fatty Acid Oxidation in Gastric Cancer. Oncogene (2019) 38:4637-54. doi: 10.1038/s41388-019-0747-0

17. Zhang F, Wang H, Yu J, Yao X, Yang S, Li W, et al. LncRNA CRNDE Attenuates Chemoresistance in Gastric Cancer via SRSF6-Regulated Alternative Splicing of PICALM. Mol Cancer (2021) 20:6. doi: 10.1186/ s12943-020-01299-y

18. Chen J, Liu L, Wei G, Wu W, Luo H, Yuan J, et al. The Long Noncoding RNA ASNR Regulates Degradation of Bcl-2 mRNA Through its Interaction With AUF1. Sci Rep (2016) 6:32189. doi: 10.1038/srep32189

19. Yuan J, Yue H, Zhang M, Luo J, Liu L, Wu W, et al. Transcriptional Profiling Analysis and Functional Prediction of Long Noncoding RNAs in Cancer. Oncotarget (2016) 7:8131-42. doi: 10.18632/oncotarget.6993

20. Tang Q, Chen J, Di Z, Yuan W, Zhou Z, Liu Z, et al. TM4SF1 Promotes EMT and Cancer Stemness via the Wnt/ß-Catenin/SOX2 Pathway in Colorectal Cancer. J Exp Clin Cancer Res (2020) 39:232. doi: 10.1186/s13046-020-01690-Z

21. Giulietti M, Righetti A, Principato G, Piva F. LncRNA Co-Expression Network Analysis Reveals Novel Biomarkers for Pancreatic Cancer. Carcinogenesis (2018) 39:1016-25. doi: 10.1093/carcin/bgy069

22. Zheng X, Wang X, Zheng L, Zhao H, Li W, Wang B, et al. Construction and Analysis of the Tumor-Specific mRNA-miRNA-lncRNA Network in Gastric Cancer. Front Pharmacol (2020) 11:1112. doi: 10.3389/fphar.2020.01112

23. Guo Y, Wang Y, Ma Y, Chen G, Yue P, Li Y. Upregulation of IncRNA SUMO1P3 Promotes Proliferation, Invasion and Drug Resistance in Gastric Cancer Through Interacting With the CNBP Protein. RSC Adv (2020) 10:6006-16. doi: 10.1039/C9RA09497K

24. Li Y, Lu L, Wu X, Li Q, Zhao Y, Du F, et al. The Multifaceted Role of Long Non-Coding RNA in Gastric Cancer: Current Status and Future Perspectives. Int J Biol Sci (2021) 17:2737-55. doi: 10.7150/ijbs.61410

25. Le K, Guo H, Zhang Q, Huang X, Xu M, Huang Z, et al. Gene and lncRNA CoExpression Network Analysis Reveals Novel ceRNA Network for Triple-Negative Breast Cancer. Sci Rep (2019) 9:15122. doi: 10.1038/s41598-019-51626-7

26. Qian J, Yang J, Liu X, Chen Z, Yan X, Gu H, et al. Analysis of lncRNA-mRNA Networks After MEK1/2 Inhibition Based on WGCNA in Pancreatic Ductal Adenocarcinoma. J Cell Physiol (2020) 235:3657-68. doi: 10.1002/jcp.29255

27. Li N, Ouyang Y, Chen S, Peng C, He C, Hong J, et al. Integrative Analysis of Differential lncRNA/mRNA Expression Profiling in Helicobacter Pylori Infection-Associated Gastric Carcinogenesis. Front Microbiol (2020) 11:880. doi: 10.3389/fmicb.2020.00880

28. Zhang C, Wu W, Zhu H, Yu X, Zhang Y, Ye X, et al. Knockdown of Long Noncoding RNA CCDC144NL-AS1 Attenuates Migration and Invasion Phenotypes in Endometrial Stromal Cells From Endometriosis $\dagger$. Biol Reprod (2019) 100:939-49. doi: 10.1093/biolre/ioy252

29. He J, Guan J, Liao S, Wu Z, Liu B, Mo H, et al. Long Noncoding RNA CCDC144NL-AS1 Promotes the Oncogenicity of Osteosarcoma by Acting as a Molecular Sponge for microRNA-490-3p and Thereby Increasing HMGA2 Expression. Oncol Targets Ther (2021) 14:1-13. doi: 10.2147/OTT.S280912

30. Zhang Y, Zhang H, Wu S. LncRNA-CCDC144NL-AS1 Promotes the Development of Hepatocellular Carcinoma by Inducing WDR5 Expression via Sponging miR-940. J Hepatocell Carcinoma (2021) 8:333-48. doi: 10.2147/ JHC.S306484 
31. Zhang L, Chi B, Chai J, Qin L, Zhang G, Hua P, et al. LncRNA CCDC144NLAS1 Serves as a Prognosis Biomarker for Non-Small Cell Lung Cancer and Promotes Cellular Function by Targeting miR-490-3p. Mol Biotechnol (2021) 63:933-40. doi: 10.1007/s12033-021-00351-6

32. Liu AN, Qu HJ, Yu CY, Sun P. Knockdown of LINC01614 Inhibits Lung Adenocarcinoma Cell Progression by Up-Regulating miR-217 and DownRegulating FOXP1. J Cell Mol Med (2018) 22:4034-44. doi: 10.1111/ jcmm. 13483

33. Vishnubalaji R, Shaath H, Elkord E, Alajez NM. Long Non-Coding RNA (lncRNA) Transcriptional Landscape in Breast Cancer Identifies LINC01614 as Non-Favorable Prognostic Biomarker Regulated by Tgf $\beta$ and Focal Adhesion Kinase (FAK) Signaling. Cell Death Discov (2019) 5:109. doi: 10.1038/s41420-019-0190-6

34. Wang Y, Song B, Zhu L, Zhang X. Long non-Coding RNA, LINC01614 as a Potential Biomarker for Prognostic Prediction in Breast Cancer. PeerJ (2019) 7:e7976. doi: 10.7717/peerj.7976

35. Wang H, Wu J, Guo W. SP1-Mediated Upregulation of IncRNA LINC01614 Functions a ceRNA for miR-383 to Facilitate Glioma Progression Through Regulation of ADAM12. Oncol Targets Ther (2020) 13:4305-18. doi: 10.2147/ OTT.S242854
36. Cai Q, Zhao X, Wang Y, Li S, Wang J, Xin Z, et al. LINC01614 Promotes Osteosarcoma Progression via miR-520a-3p/SNX3 Axis. Cell Signal (2021) 83:109985. doi: 10.1016/j.cellsig.2021.109985

Conflict of Interest: The authors declare that the research was conducted in the absence of any commercial or financial relationships that could be construed as a potential conflict of interest.

Publisher's Note: All claims expressed in this article are solely those of the authors and do not necessarily represent those of their affiliated organizations, or those of the publisher, the editors and the reviewers. Any product that may be evaluated in this article, or claim that may be made by its manufacturer, is not guaranteed or endorsed by the publisher.

Copyright (c) 2022 Sheng, Zhou, Cao and Zhong. This is an open-access article distributed under the terms of the Creative Commons Attribution License (CC BY). The use, distribution or reproduction in other forums is permitted, provided the original author(s) and the copyright owner(s) are credited and that the original publication in this journal is cited, in accordance with accepted academic practice. No use, distribution or reproduction is permitted which does not comply with these terms. 